\title{
Calculating Summary Measures of Unimodal Response Curves by Means of Nonlinear Regression Models
}

\author{
RALF BENDER* \\ Department of Metabolic Diseases and Nutrition, Heinrich-Heine-University of Düsseldorf, P.O. Box 101007, D-40001 Diisseldorf, \\ Germany
}

(Received I0 March 1998; In final form 8 January 1999)

\begin{abstract}
In biomedical research summary measures such as the curve maximum $\left(C_{\max }\right)$ are frequently used to describe and analyze unimodal response curves. However, if the true curve is perturbed by autocorrelated noise the calculation of summary measures from raw data can be arbitrary and misleading due to high peaks produced by the correlated errors. A possible solution is to fit suitable nonlinear functions to the response curves and estimate the summary measures from these functions. In this paper formulas are derived providing a way to estimate important summary measures of unimodal curves by means of the parameters of the lognormal function. The method is illustrated by application to pharmacodynamic data.
\end{abstract}

Keywords: Response curves, summary measures, nonlinear regression analysis, autocorrelated errors, lognormal function

\section{INTRODUCTION}

In biomedical research summary measures are frequently used to describe and analyze response curves (Matthews et al., 1990; Everitt, 1995). In the case of unimodal curves important summary measures are the curve maximum $\left(C_{\max }\right)$, the time to the curve maximum $\left(t_{\max }\right)$, and the area under the curve $(A U C)$. To calculate $A U C$ a time interval must be specified to which the area refers. In pharmacokinetics the area corresponding to an interval $[0, T]$, where $T$ is any time point (frequently the last sampling time), is called the partial area $\left(A U C_{0-T}\right)$, while the area from 0 to infinity $\left(A U C_{0-\infty}\right)$ is called the total area under the curve (Wagner and Ayres, 1977). In some applications, e.g. the description of the time action-profiles of insulin preparations, the time points to the half of the curve maximum $\left(t_{\text {low }}, t_{\text {up }}\right)$ are also of interest (Heinemann et al., 1996; 1997; 1998).

It is not always possible to calculate summary measures of observed response curves simply from raw data. For the estimation of summary measures such as $t_{\text {low }}$ and $t_{\text {up }}$ a continuous curve is required. Hence, at least an interpolation of the measured curve at discrete time points is needed. For the calculation of $A U C_{0-\infty}$ a pharmacokinetic or mathematical model is required allowing an estimation

*Tel: +49(0)211 81-13981; Fax:+49(0)21181-14294; E-mail: bender@uni-duesseldorf.de 
of the $A U C$ beyond the last sampling time. Some special problems are induced by autocorrelated time series errors. If the true curve is perturbed by autocorrelated noise the calculation of summary measures from raw data can be quite misleading. Autocorrelated errors can produce artificial high peaks so that the observed curve maximum would substantially overestimate $C_{\max }$.

A possible solution to all these problems is given by fitting suitable regression functions to the response curves. The framework of nonlinear regression allows the consideration of autocorrelated time series errors. The fitted regression function represents a continuous curve so that even summary measures such as $t_{\text {low }}, t_{\text {up }}$, and $A U C_{0-\infty}$ can be calculated from the fitted regression function.

In this paper a review of nonlinear regression techniques allowing autocorrelated errors is given. Especially, the lognormal function is used to describe unimodal curves occuring in pharmacodynamic studies of insulin preparations (Bender and Heinemann, 1994; 1995). It is shown that the summary measures mentioned above can be calculated as direct functions from the parameters of the lognormal function. The method is illustrated by estimating summary measures of time-action profiles of a regular human insulin preparation.

\section{NONLINEAR REGRESSION WITH CORRELATED ERRORS}

Let $y_{t}$ denote the measurement of a considered response curve at time $t$. Due to the reasons discussed above, summary measures of the response curve cannot be calculated adequately from the raw data $y_{t}$. Instead a nonlinear regression function should be fitted to the response curve. The general nonlinear model is given by

$$
y_{t}=f(t, \theta)+u_{t}
$$

where $f$ is a nonlinear function of $t$ with parameter vector $\theta$ and $u_{t}$ are random errors, called residuals (Seber and Wild, 1989). If $f$ is a linear function, e.g. $f(t)=\alpha+\beta t$, model (1) reduces to the simple linear regression model. Nonlinear models are more difficult to specify and estimate than linear models. Instead of simply listing explanatory factors, a suitable nonlinear function must be specified. Additionally, adequate starting values for the regression coefficients are required for the iterative estimation procedure.

In standard nonlinear regression it is assumed that the residuals $u_{t}$ are independent and identically distributed (i.i.d.). In this case nonlinear ordinary least squares (OLS) can be used for parameter estimation. However, in practice the i.i.d. assumption is frequently violated. The most important generalizations are heteroscedastic errors (Beal and Sheiner, 1988), i.e. unequal error variances, and autocorrelated errors (Seber and Wild, 1989), i.e. the errors $u_{t}$ represent a time series (Wei, 1990). Both violations require special considerations and estimation procedures. Since the case of heteroscedastic errors is treated in detail in the pharmacokinetic literature (Sheiner, 1984; 1985; 1986; Giltinan and Ruppert, 1989) we concentrate on autocorrelated errors.

The usual method to take autocorrelated errors of a regression model into account is to assume that the errors $u_{t}$ follow a stationary autoregressive movingaverage (ARMA) process (Seber and Wild, 1989). One important subclass of ARMA models is given by the autoregressive (AR) processes of order $p$

$$
u_{t}=\sum_{j=1}^{p} \varphi_{j} u_{t-j}+\varepsilon_{t}
$$

where $\varepsilon_{t}$ are i.i.d. with mean zero and variance $\sigma_{\varepsilon}^{2}$, $\varphi_{j}$ for $j=1, \ldots, p$ are the AR parameters, and $t=$ $1, \ldots, n$ are equally spaced time points. In practice, a value for the order $p$ must be chosen. For this, the Box-Jenkins method (Wei, 1990) can be applied. A brief overview of this method is given by Seber and Wild (1989). In practice, the use of low-order $\operatorname{AR}(p)$ models (i.e. $p \leq 3$ ) is often sufficient, as $\mathrm{AR}(2)$ or $\mathrm{AR}(3)$ models produce at least reasonable approximations to the true correlation structure of the errors $u_{\ell}$.

A number of procedures are available for parameter estimation in model (1) assuming an $\operatorname{AR}(p)$ model (2) for the errors $u_{t}$. Most procedures are 
based upon nonlinear generalized least squares (GLS) which takes the correlation of the errors into account. As the corresponding covariance matrix has to be estimated, all approaches represent multistep procedures, frequently by starting with nonlinear OLS. An overview of these estimation procedures with technical details is given by Seber and Wild (1989). A comfortable software to fit nonlinear regression models with ARMA errors is given by the SAS/ETS ${ }^{\circledR}$ procedure MODEL (SAS, 1988).

Although the presence of autocorrelated errors is one reason to estimate summary measures via nonlinear regression methods, the neglect of the autocorrelation has little impact on the estimation of the regression coefficients (Bender and Heinemann, 1995). However, the standard errors of the regression coefficients are considerably underestimated when the correlation of the errors is disregarded (Glasbey, 1980; Bender and Heinemann, 1995). Hence, if one is interested in a reliable information about the uncertainty of the estimated regression coefficients, it is desirable to take account of the autocorrelation in the estimation procedure.

\section{SUMMARY MEASURES OF LOGNORMAL CURVES}

In this paper the lognormal function is considered to describe unimodal curves occuring in pharmacodynamic studies investigating the time-action profiles of insulin preparations (Heinemann et al., 1996; $1997 ; 1998)$. The lognormal function is given by

$$
\begin{aligned}
f(t)=a t^{-1} & e^{-b[\log (t)-c]^{2}} \\
& (t>0, a, b, c>0)
\end{aligned}
$$

where $t$ is the time and $a, b, c$ are the regression coefficients. Although the lognormal function has only three parameters it is very flexible to cover a wide range of unimodal curves. This function has desirable properties to describe the time-action profiles of insulin preparations (Bender and Heinemann, $1994 ; 1995)$. Of course, in these and other applications the use of other nonlinear functions, e.g. gamma functions or polyexponentials, is possible. For each nonlinear function summary measures have to be derived separately.

In the following formulas are presented for the calculation of summary measures based upon the lognormal function. While some of these formulas can be easily derived by means of standard calculations, others require some tricks. A step to step calculation is given in the Appendix. As all formulas represent explicit functions of the lognormal parameters, they can easily be computed by means of $\mathrm{SAS}^{\circledR}$ (SAS, 1985) or other programs containing usual mathematical functions.

The formulas for calculating summary measures from the lognormal parameters do not depend on the special estimation procedure used to fit lognormal functions. Any formulation for the structure of the residuals, which is reasonable for the data considered, is possible. However, it is assumed that a lognormal curve represents an adequate description of the data considered and that one is able to estimate the unknown lognormal parameters by means of a suitable method. The estimated lognormal parameters can then be used as basis for the calculation of summary measures.

Let $a, b, c$ be the parameters of the lognormal function (3) and let $\Phi$ denote the distribution function of the standard normal distribution, then the following formulas are valid for lognormal curves.

$$
\begin{aligned}
t_{\max } & =\exp \left(c-\frac{1}{2 b}\right) \\
C_{\mathrm{max}} & =a \exp \left(\frac{1}{4 b}-c\right) \\
t_{\mathrm{low}} & =\exp \left(c-\frac{1}{2 b}-\sqrt{\frac{\log (2)}{b}}\right) \\
t_{\mathrm{up}} & =\exp \left(c-\frac{1}{2 b}+\sqrt{\frac{\log (2)}{b}}\right) \\
A U C_{0-\infty} & =a \sqrt{\frac{\pi}{b}} \\
A U C_{0-T} & =a \sqrt{\frac{\pi}{b}} \Phi(\sqrt{2 b}(\log (T)-c))
\end{aligned}
$$




\section{EXAMPLE}

The method described is applied to pharmacodynamic data of an euglycemic glucose-clamp study. In such studies blood glucose concentration is held constant after subcutaneous injection of insulin preparations by varying glucose infusion rates (GIR) automatically be means of a Biostator. Thus, the GIR curves represent an indirect measurement of the pharmacodynamic time-action profiles of the considered insulin preparations. In the following, only two curves of a glucose-clamp study investigating the pharmacodynamics of a regular human insulin preparation (Heinemann, 1998) are analyzed for demonstrating purposes. These two curves were measured from the same individual under equal conditions.

At first, lognormal functions are fitted to the GIR curves (Figure 1). As the glucose-clamp algorithm employed by the Biostator leads to correlated errors, the residuals $u_{t}$ are modeled as autoregressive (AR) processes of order 3. Details of this estimation procedure, especially a method to find suitable starting values for the iterative algorithm, as well as the corresponding $\mathrm{SAS}^{(B)}$ code are given by Bender and Heinemann (1995). The lognormal parameters of the fitted curves are $a=2010.96$, $b=1.3817, c=5.4823$ for GIR curve 1 and $a=$ $2050.91, b=1.8797, c=5.3878$ for GIR curve 2 .

Secondly, the summary measures are calculated by using the formulas (4) to (7b). These results were compared with the summary measures calculated from the raw data (Table I). For $C_{\max }$ the maximum GIR and for $t_{\max }$ the time point of the maximum GIR were used. If the maximal GIR was reached at several time points, for $t_{\max }$ the average of the corresponding time points was used. $A U C_{0-T}$ (for $T=600$ ) was calculated by means of the trapezoidal rule. The summary measures $A U C_{0-\infty}$ and tow,up can not be calculated directly from the raw data.

Thirdly, standard errors and $95 \%$ confidence intervals for the important summary measures $t_{\max }, C_{\max }$, and $A U C_{0-\infty}$ are calculated by means of the multivariate delta method (Bender, 1996). The results are shown in Table II. The documentation of the uncertainty of the summary measures estimated from raw data is impossible.

Obviously, the GIR curves can not adequately be described by means of summary measures calculated from the raw data. While the estimates for $A U C_{0-T}$ are comparable, $C_{\max }$ is overestimated in both curves if simply the maximum GIR is used. This result is typical for GIR curves because the autocorrelation of the residuals will always produce more or less artificial high peaks. If an extremely high peak is present in the beginning or the end of the GIR curve the corresponding $t_{\max }$ value will also be misleading (GIR curve 2). The summary measures calculated from the raw data pretend a large difference between the two GIR curves. In fact, the true curves are quite similar, which is expected as they are measured from the same individual under similar conditions. What is different between the curves, is the variability of the random errors which is higher in the second curve resulting in higher peaks. This is well documented by the larger standard errors and wider confidence intervals of the summary measures of curve 2 . However, the main goal of glucose-clamp studies is to investigate the pharmacodynamic properties of the insulin preparations and not the variability and correlation structure of the errors produced by the Biostator. Hence, the main interest applies to the true GIR curves, which seems to be adequately estimated by means of the fitted lognormal curves.

\section{DISCUSSION}

The description and analysis of response curves by means of summary measures is a useful tool in biomedical and pharmaceutical research. The method of calculating summary measures via nonlinear regression models has several advantages.

Firstly, the framework of nonlinear regression permits the consideration of various error structures, e.g. autocorrelated errors. This is important, because autocorrelated noise can lead to inappropriate estimates of the summary measures if the raw data are used for estimation. Secondly, it is possible to 


\section{GIR Curve 1}

$\operatorname{GIR}(\mathrm{mg} / \mathrm{kg} / \mathrm{min})$

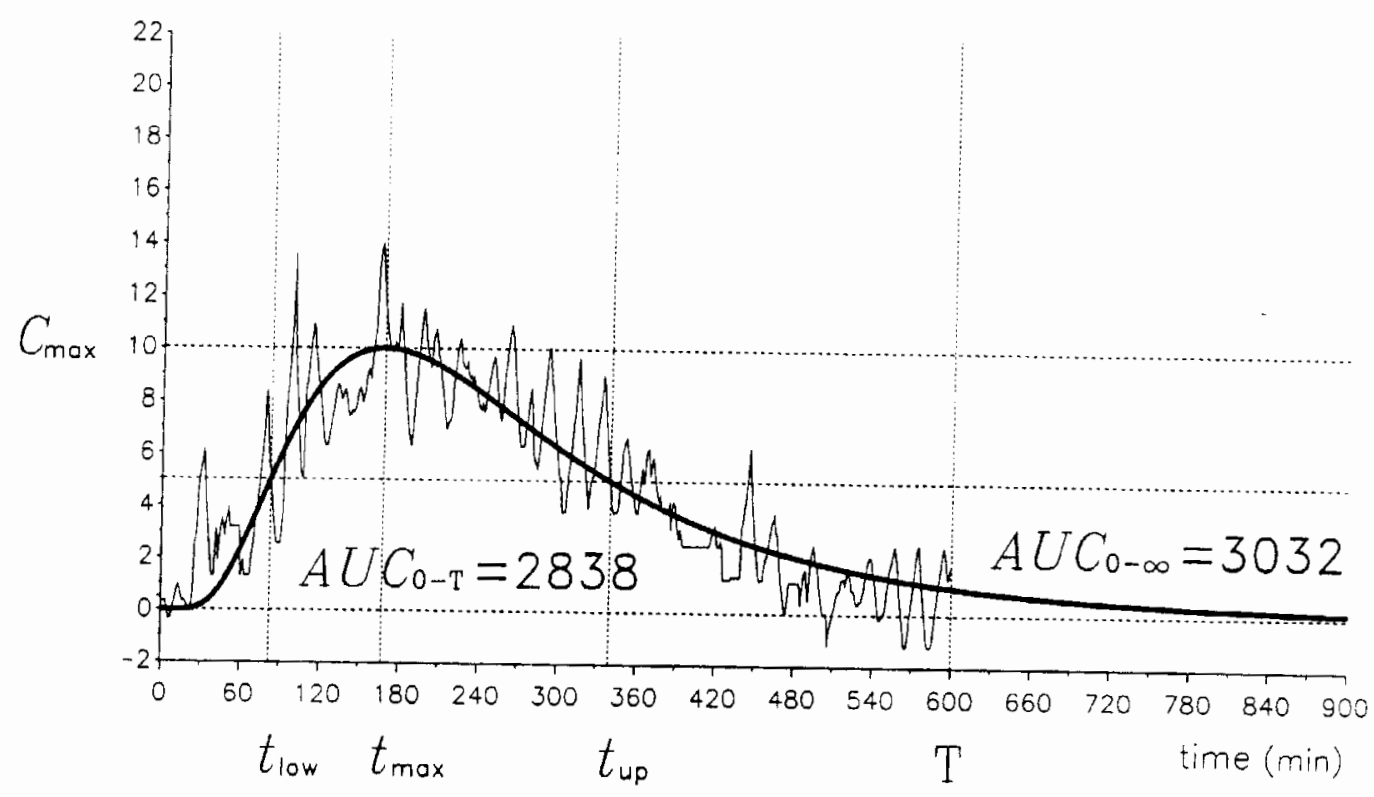

GIR Curve 2

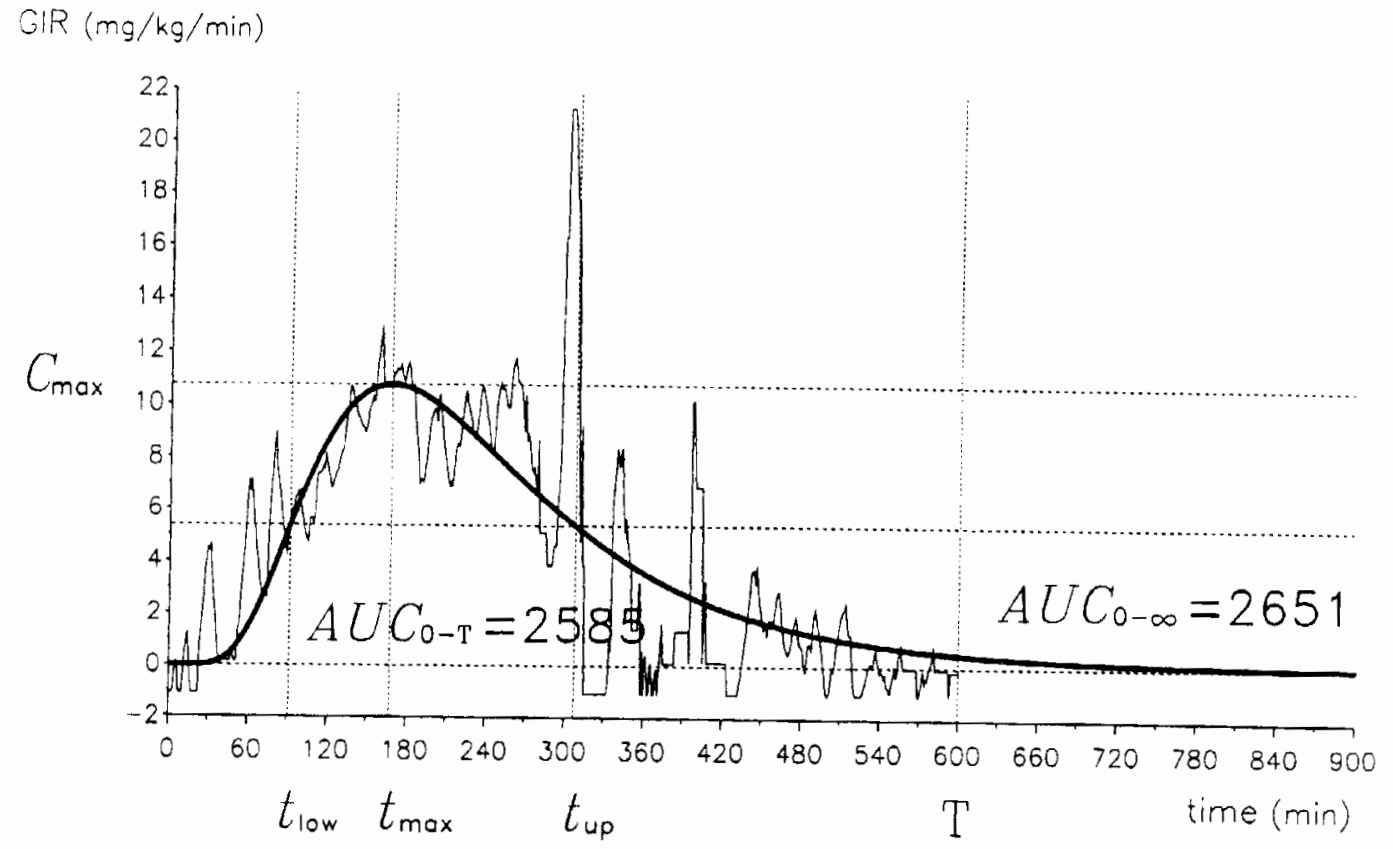

FIGURE I Summary measures of two time-action profiles (GIR curves) of a regular human insulin (thin line) calculated from the parameters of fitted lognormal functions (thick line). 
TABLE I Summary measures of two GIR curves calculated from fitted lognormal curves and from raw data

\begin{tabular}{lccccc}
\hline Summary Measure & \multicolumn{2}{c}{ GIR curve 1 } & \multicolumn{2}{c}{ GlR curve 2 } \\
\cline { 2 - 3 } & Lognormal Curve & Raw Data & & Lognormal Curve & Raw Data \\
\hline$t_{\max }(\mathrm{min})$ & 167.4 & 165.0 & 167.6 & 301.5 \\
$C_{\max }(\mathrm{mg} / \mathrm{min} / \mathrm{kg})$ & 10.02 & 13.99 & 10.71 & 21.27 \\
$t_{\text {low }}(\mathrm{min})$ & 82.4 & - & 91.3 & - \\
$t_{\text {up }}(\mathrm{min})$ & 339.9 & - & 307.7 & - \\
$A U C_{0-\infty}(\mathrm{mg} / \mathrm{kg})$ & 3032.3 & - & 2651.4 & - \\
$A U C_{0-T}(\mathrm{mg} / \mathrm{kg})$ & 2837.7 & 2860.2 & 2584.6 & 2550.3 \\
\hline
\end{tabular}

TABLE II Standard errors and 95\% confidence intervals of the summary measures estimated from fitted lognormal curves

\begin{tabular}{lccccccc}
\hline Summary & \multicolumn{3}{c}{ GIR curve 1} & & \multicolumn{3}{c}{ GIR curve 2 } \\
\cline { 2 - 3 } & $\begin{array}{c}\text { Measure } \\
\text { estimate }\end{array}$ & $\begin{array}{c}\text { Standard } \\
\text { error }\end{array}$ & $\begin{array}{c}95 \% \text { confidence } \\
\text { interval }\end{array}$ & & $\begin{array}{c}\text { Point } \\
\text { estimate }\end{array}$ & $\begin{array}{c}\text { Standard } \\
\text { error }\end{array}$ & $\begin{array}{c}95 \% \text { confidence } \\
\text { interval }\end{array}$ \\
\hline$t_{\max }$ & 167.4 & 5.17 & $157.3-177.5$ & & 167.6 & 13.93 & $140.3-194.9$ \\
$C_{\max }$ & 10.02 & 0.39 & $9.25-10.79$ & & 10.71 & 1.36 & $8.05-13.37$ \\
$A U C_{0-\infty}$ & 3032.3 & 138.1 & $27621-3303$ & & 2651.4 & 370.2 & $1925-3377$ \\
\hline
\end{tabular}

calculate summary measures which require a continuous curve such as the time points to the half of the curve maximum. Thirdly, summary measures for which information beyond the last sampling time is needed such as the total area under the curve can be estimated. Fourthly, the information of curves containing a large number of time points can be comprised in few regression coefficients. A lognormal curve is sufficiently described by only three parameters. Finally, as the summary measures are nonlinear functions of the regression coefficients it is possible to estimate standard errors and confidence intervals of the summary measures by means of the delta method in order to describe the precision of the estimation (Bender, 1996).

The adequacy of the presented approach depends strongly on the adequacy of the regression functions fitted to the response curves. If the regression model is misspecified all results are questionable. Hence, before summary measures are calculated from regression coefficients, the adequacy of the regression function should be investigated very carefully. In cases where a nonlinear function to describe the response curve adequately is not available, smoothing methods are an alternative (Diggle and Hutchinson, 1989).

The fitting of nonlinear functions can be laborious, especially if no suitable starting values for the iterative estimation procedure are available. For the fitting of lognormal functions with autocorrelated errors a $S_{A S}{ }^{(\mathbb{R}}$ program is available, which includes a method for finding suitable starting values of the lognormal parameters automatically (Bender and Heinemann, 1995).

In conclusion, summary measures of unimodal curves can be calculated efficiently by means of nonlinear regression models. This approach permits the consideration of autocorrelated errors, makes it possible to estimate summary measures which are not directly available from the raw data, represents an enormous data reduction, and allows the investigation of the precision of the estimated summary measures.

\section{APPENDIX}

To derive the formulas (4) to (7b) some equations and lemmas are required. The lognormal function 
and the first derivative are given by

$$
\begin{array}{r}
f(t)=a t^{-1} \exp \left(-b[\log (t)-c]^{2}\right) \\
(t>0, a, b, c>0) \\
f^{\prime}(t)=-a t^{-2}[2 b \log (t)-2 b c+1] \\
\times \exp \left(-b[\log (t)-c]^{2}\right)
\end{array}
$$

The integral of a quadratic exponential function can be solved by (Bronštein and Semendjajew, 1981)

$$
\int_{0}^{\infty} \exp \left(-\omega^{2} x^{2}\right) d x=\frac{\sqrt{\pi}}{2 \omega}
$$

The density function $l(y)$ of the lognormal distribution with parameters $\mu$ and $\sigma^{2}$ [denoted in the following by $\left.\mathrm{LN}\left(\mu, \sigma^{2}\right)\right]$ is given by

$$
l(y)=\frac{1}{\sqrt{2 \pi \sigma^{2}}} y^{-1} \exp \left(-\frac{1}{2 \sigma^{2}}[\log (y)-\mu]^{2}\right)
$$

By defining $\alpha=a \sqrt{\pi / b}, \quad a=1 / \sqrt{2 \pi \sigma^{2}}, \quad b=$ $1 /\left(2 \sigma^{2}\right)$, and $c=\mu$ we get the relation

$$
f(t)=\alpha l(t)
$$

If $X$ is a random variable with distribution function $F_{X}$ and if $Y=g(X)$ then the distribution function of $Y$ can be computed by

$$
F_{Y}(y)=F_{X}\left(g^{-1}(x)\right)
$$

As the log of a lognormal distributed variable yields a normal distributed variable it follows that

$$
F_{\mathrm{LN}\left(\mu, \sigma^{2}\right)}(y)=\Phi\left(\frac{\log (y)-\mu}{\sigma}\right)
$$

where $\Phi$ denotes the distribution function of the standard normal distribution.

The formulas (4) to ( $7 \mathrm{~b})$ can now be derived as follows.

1)

$$
\begin{aligned}
t_{\max }: f^{\prime}(t) & =0 \stackrel{(\mathrm{A} 2)}{\Leftrightarrow} 2 b \log (t)-2 b c+1=0 \\
& \Rightarrow t_{\max }=\exp \left(c-\frac{1}{2 b}\right)
\end{aligned}
$$

2)

$$
C_{\max }: C_{\max }=f\left(t_{\max }\right) \stackrel{(\mathrm{A} 1)}{=} a \exp \left(\frac{1}{4 b}-c\right)
$$

3)

$$
\begin{aligned}
& t_{\mathrm{low}, \mathrm{up}}: f(t)=\frac{1}{2} C_{\mathrm{max}} \\
& \Leftrightarrow a t^{-1} \exp \left(-b[\log (t)-c]^{2}\right) \\
& \quad=\frac{1}{2} a \exp \left(\frac{1}{4 b}-c\right) \\
& \Leftrightarrow \log ^{2}(t)+\frac{1-2 b c}{b} \log (t)+c^{2} \\
& +\frac{1}{4 b^{2}}-\frac{c}{b}-\frac{\log (2)}{b}=0 \\
& \Rightarrow t_{\mathrm{low}, \mathrm{up}}=\exp \left(c-\frac{1}{2 b} \pm \sqrt{\frac{\log (2)}{b}}\right)
\end{aligned}
$$

4) $A U C_{0-\infty}$ : Let $z=g(t)=\log (t)-c, g^{\prime}(t)=t^{-1}$, and $h(x)=\exp \left(-b x^{2}\right)$ then it follows from the substitution rule

$$
\begin{aligned}
A U C_{0-\infty} & =\int_{0}^{\infty} f(t) d t \\
& =a \int_{0}^{\infty} t^{-1} \exp \left(-b[\log (t)-c]^{2}\right) d t \\
& =a \int_{0}^{\infty} h(g(t)) \cdot g^{\prime}(t) d t \\
& =a \int_{g(0)}^{g(\infty)} h(z) d z \\
& =a \int_{-\infty}^{+\infty} \exp \left(-b z^{2}\right) d z^{(\mathrm{A} 3)}=a \sqrt{\frac{\pi}{b}}
\end{aligned}
$$

5)

$$
\begin{aligned}
& A U C_{0-T}: A U C_{0-T}=\int_{0}^{T} f(t) d t \\
& \stackrel{\text { (A4) }}{=} \alpha \int_{0}^{T} l(t) d t=\alpha F_{\mathrm{LN}}\left(\mu, \sigma^{2}\right)(T) \\
& \stackrel{\text { (A5) }}{=} \alpha \Phi\left(\frac{\log (T)-\mu}{\sigma}\right) \\
& \stackrel{\text { (A4) }}{=} a \sqrt{\frac{\pi}{b}} \Phi(\sqrt{2 b}(\log (T)-c))
\end{aligned}
$$

\section{Acknowledgements}

I thank Dr. Lutz Heinemann for providing the GIR data used in the example. 


\section{References}

Beal, S. L. and Sheiner, L. B. (1988). Heteroscedastic nonlinear regression. Technometrics, 30, 327-338.

Bender, R. (1996). Calculating confidence intervals for summary measures of individual curves via nonlinear regression. Inter national Journal of Biomedical Computing, 41, 13-18.

Bender, R. and Heinemann, L. (1994). Description of peaked curves by means of cxponential functions. In: Pöppl, S. J., Lipinski, H.-G. and Mansky. T. (eds.): Medizinische Informatik: Ein integrierender Teil arztunterstützender Technologien. Proc. 38. Jahrestagung der GMDS. MMV Medizin Verlag, München, pp. $359-365$.

Bender, R. and Heinemann, L. (1995). Fitting nonlinear regression models with correlated errors to individual pharmacodynamic data using SAS software. Joumal of Pharmacokinetics and Biopharmaceutics, 23, 87-100.

Bronštein, I. N. and Semendjajew, K. A. (1981). Taschenbuch der Mathematik (21. Auf.). Teubner, Leipzig, p. 66.

Diggle. P. J. and Hutchinson, M. F. (1989). On spline snoothing with autocorrelated errors. Australian Journal of Statistics, 31 $166-182$.

Everitt. B. S. (1995). The analysis of repeated measures: a practical review with examples. Statistician, 44, 113-135.

Giltinan, D. M. and Ruppert, D. (1989). Fitting heteroscedastic regression models to individual pharmacokinetic data using standard statistical software. Journal of Pharmacokinetics and Biophurmaceutics, 17, 601-614.

Glasbey, C. A. (1980). Nonlinear regression with autoregressive time series errors. Biometrics, 36, 135-140.

Heinemann, L., Kapitza, C., Starke, A. A. R. and Heise, T. (1996). Time-action profile of the insulin analogue B28Asp Diabetic Medicine, 13, 683-684.
Heinemann, L., Traut, T. and Heise, T. (1997). Time-action profile of inhaled insulin. Diabetic Medicine, 14, 63--72.

Heinemann, L., Weyer, C., Rauhaus, M., Heinrichs, S., and Heise, T. (1998). Variability of the metabolic effect of solubje insulin and the rapid-acting insulin analog insulin aspart. Diabetes Care, 21, 1910-1914.

Matthews, J. N. S.. Altman, D. G., Campbell, M. J. and Royston, P. (1990). Analysis of serial measurements in medical research, British Medical Journul, 300, 230-235.

SAS (1985). SAS/IML ${ }^{(8)}$ User's Guide, Version 5 Edition. SAS Institute Inc., Cary, NC.

SAS (1988). SAS/ETS ${ }^{\circledR}$ User's Guide, Version 6, First Edition. SAS Institute Inc., Cary, NC, 1988.

Seber, G. A. F, and Wild, C. J. (1989). Nonlinear Regression. Wiley, New York

Sheiner, L. B. (1984). Analysis of pharmacokinetic data using parametric models. I. Regression models. Journal of Pharmacokinetics and Biopharmarceutics, 12, 93-117.

Sheiner, L. B. (1985). Analysis of pharmacokinetic data using parametric models. II. Point estimates of an individual's parameters. Journal of Pharmacokinetics and Biopharmarceutics, 13, 515-540.

Sheiner, L. B. (1986). Analysis of pharmacokinetic data using parametric models. III. Hypothesis testing and confidence intervals. Journal of Pharmacokinetics and Biopharmarceutics, 14, 539-555.

Wagner, J. G. and Ayres, J. W. (1977). Bioavailability assessment: methods to estimate total area $\left(A U C_{0-x}\right.$ and total amount excreted $\left(A^{\infty}\right)$ and importance to blood and urine sampling scheme with application to digoxin. Journal of Pharmacokinetics and Biopharmarceutics, 5, 533-557.

Wei, W. W. S. (1990). Time Series Analysis. Univariate and Multivariate Methods. Addison-Wesley, Redwood City. 


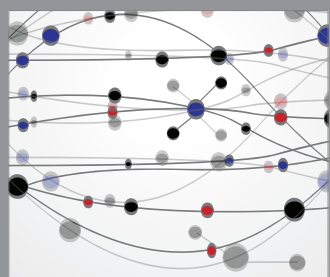

The Scientific World Journal


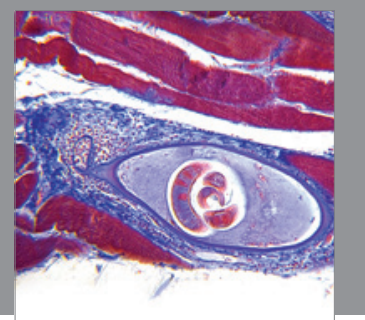

Gastroenterology

Research and Practice


\section{Hindawi}

Submit your manuscripts at

http://www.hindawi.com
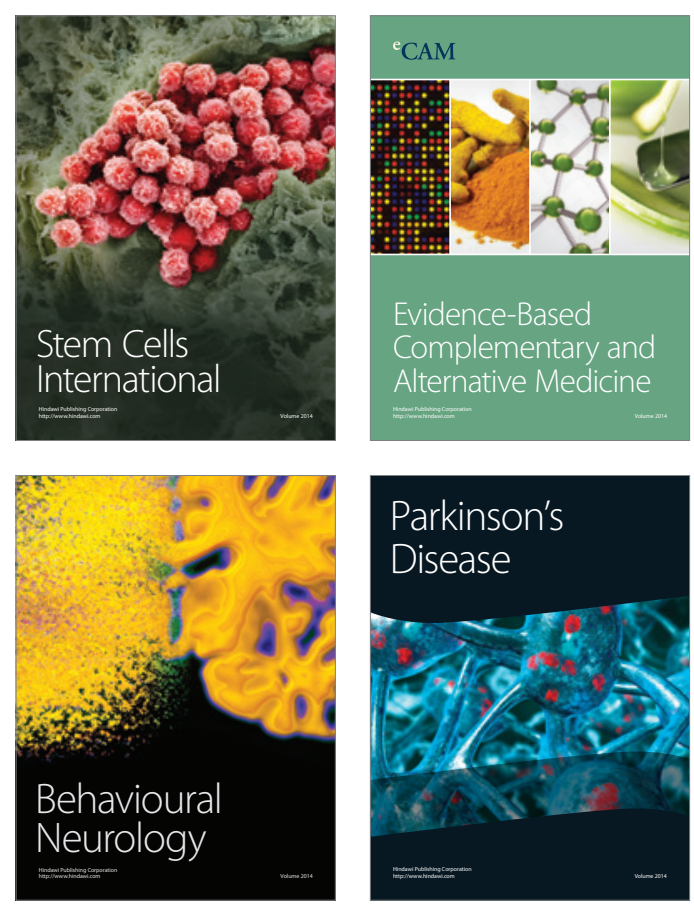



Journal of
Diabetes Research

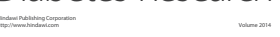

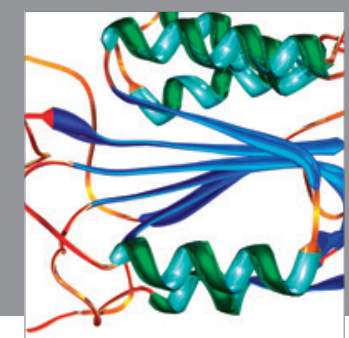

Disease Markers
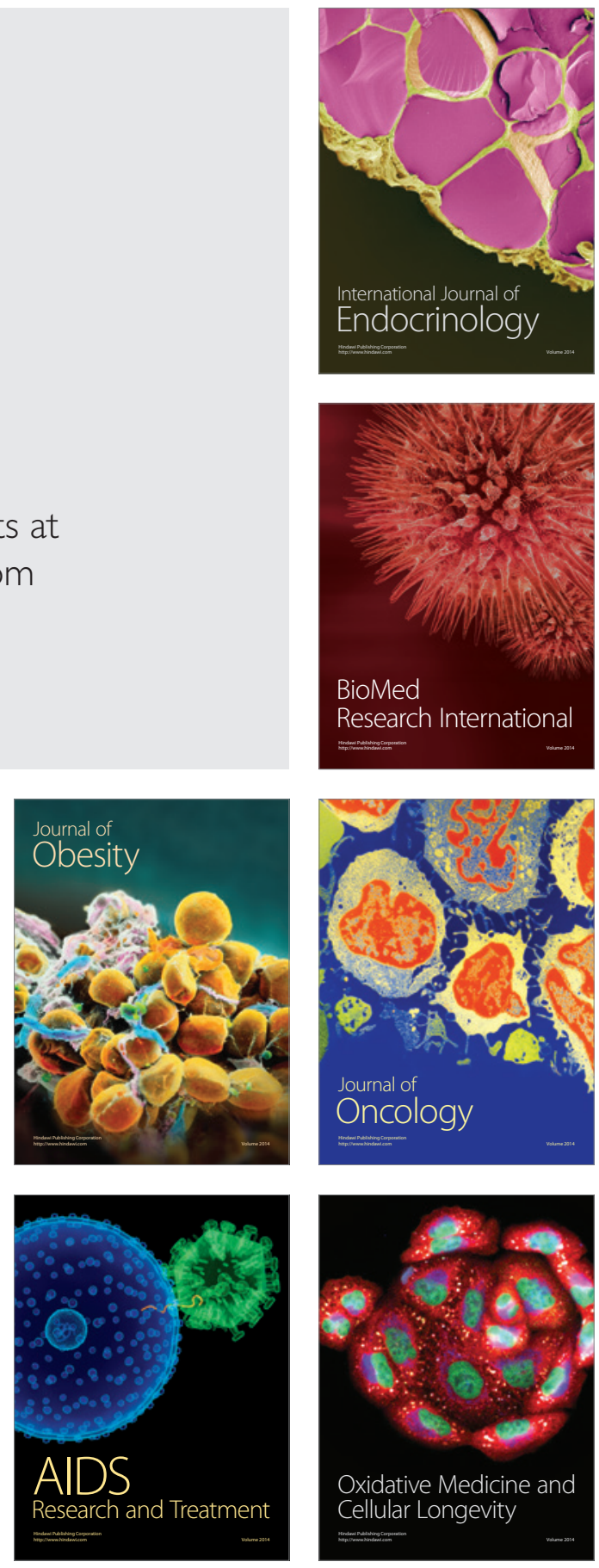